\title{
Predicting Recurrence in Endometrial Cancer Based on a Combination of Classical Parameters and Immunohistochemical Markers
}

This article was published in the following Dove Press journal:

Cancer Management and Research

\section{Peng Jiang* \\ Jin Huang $\mathbb{D}^{*}$ \\ Ying Deng \\ Jing $\mathrm{Hu}$ \\ Zhen Huang \\ Mingzhu Jia \\ Jiaojiao Long \\ Zhuoying $\mathrm{Hu}$}

Department of Gynecology, First Affiliated Hospital of Chongqing Medical University, Chongqing, People's Republic of China

*These authors contributed equally to this work
Correspondence: Zhuoying Hu Department of Gynecology, First Affiliated Hospital of Chongqing Medical University, Chongqing, People's Republic of China

Tel $+86238901-1092$

Fax +862389011082

Email huzhuoying@sina.com
Objective: The aim of this study was to establish a nomogram to predict the recurrence of endometrial cancer (EC) by immunohistochemical markers and clinicopathological parameters and to evaluate the discriminative power of this model.

Methods: The data of 473 patients with stages I-III endometrial cancer who had received primary surgical treatment between October 2013 and May 2018 were randomly split into two sets: a training cohort and a validation cohort at a predefined ratio of 7:3. Univariate and multivariate Cox regression analysis of screening prognostic factors were performed in the training cohort $(n=332)$ to develop a nomogram model for EC-recurrence prediction, which was further evaluated in the validation cohort $(n=141)$.

Results: Univariate analysis found that FIGO stage, histological type, histological grade, myometrial invasion, cervical stromal invasion, postoperative adjuvant treatment, and four immunohistochemical markers (Ki67, ER, PR, and p53) were associated with recurrence in EC. Multivariate analysis showed that FIGO stage, histological type, ER, and p53 were superior parameters to generate the nomogram model for recurrence prediction in EC. Recurrence-free survival was better predicted by the proposed nomogram, with a $\mathrm{C}$-index value of 0.79 (95\% CI $0.66-0.92)$ in the validation cohort.

Conclusion: This nomogram model involving immunohistochemical markers can better predict recurrence in FIGO stages I-III EC.

Keywords: classical parameters, immunohistochemical markers, endometrial cancer, predicting model, recurrence

\section{Introduction}

The 5-year overall survival rate of endometrial cancer (EC) is about $80 \%{ }^{1}$ Though early diagnosis could be an important factor contributing to such a high overall survival rate, if tumor type or other related factors are taken into consideration, the survival rate varies significantly. ${ }^{2,3}$ Relapse is still one of the leading causes of EC mortality. ${ }^{4}$ Therefore, it would be of great help for a model to predict precisely the of EC recurrence, which would be better for identifying patients to be put onto a more proper scheme of treatment and follow-up. Most of the current models for predicting recurrence in EC are based on classical clinicopathological parameters. ${ }^{5}$ The most recent study by Ouldamer et al developed a nomogram model based on age, International Federation of Gynecology and Obstetrics (FIGO) stage, histological type and grade, lymphovascular space invasion, and surgical nodal staging to predict prognosis of patients with stages I-III EC with good discrimination. ${ }^{4}$ Immunohistochemical markers have been widely applied in 
pathological reports, and have already became an important indicator for prognosis in EC and other tumors. ${ }^{6,7}$ In breast cancer, the prognostic prediction value has been optimized by adding four immunohistochemical markers - ER, PR, HER2, and Ki67 - to the traditional model.. ${ }^{8}$ Therefore, it is of great necessity to incorporate immunohistochemical markers into the traditional models and develop a more precise prediction model for recurrence in EC to facilitate individualized treatment for patients.

\section{Methods}

\section{Study Population}

We conducted a retrospective analysis of the data of 537 patients with EC who received primary surgical treatment between October 2013 and May 2018 at the First Affiliated Hospital of Chongqing Medical University. Inclusion criteria were patients diagnosed with stages I-III EC according to the updated staging system of FIGO, ${ }^{9}$ complete case records, including age, body-mass index (BMI), comorbidities (eg, hypertension or diabetes), surgical records, pathological results (histological type, grade, depth of myometrial invasion, and cervical stromal invasion), four immunohistochemical markers (Ki67, ER, PR, and p53), and postoperative treatment. Exclusion criteria were patients not following the routine standardized surgical treatment, ${ }^{4}$ pathological analysis indicated endometrial sarcoma, Those with other malignancies, having received chemotherapy or radiotherapy before surgery, and patients who failed to adhere to postoperative and follow-up schemes. ${ }^{10}$

\section{Treatment and Follow-Up}

All patients had received standard surgical treatment, including total hysterectomy with bilateral salpingo-oophorectomy, with or without nodal staging (sentinel lymph node \pm pelvic \pm para-aortic lymphadenectomy). ${ }^{4}$ Postoperative treatment, including chemotherapy or radiotherapy, was determined by a multidisciplinary team as per the 2013 ESMO guidelines. ${ }^{11}$ Paclitaxel and nedaplatin had been used in chemotherapy at a dose of $135-175 \mathrm{mg} / \mathrm{m}^{2}$ intravenously and $80-100 \mathrm{mg} / \mathrm{m}^{2}$, respectively. Follow-up visits were performed every 3 months for the first 2 years, every 6 months for the following 3 years, and once a year thereafter until 5 years after operation. Each follow-up included at least regular physical examinations plus necessary imaging or blood tests. Except for some patients who died due to recurrence or other disease during follow-up, all patients had follow-up $>1$ year.

\section{Recurrence}

Recurrence was considered if lesions were confirmed by physical examination, histological examination, or images, including CT, MRI, ultrasonography, bone scintigraphy, FDG-PET, or specific X-rays. ${ }^{12}$ Recurrence-free survival (RFS) was defined as time between date of complete surgical removal of the malignancy and either date of histologically or radiologically confirmed recurrence. ${ }^{13}$ Overall survival was defined as time from primary surgery to death. ${ }^{12}$

\section{Histology and Immunohistochemistry}

All postoperative specimens were processed in the Department of Pathology as per lab protocols reported previously. ${ }^{14}$ Briefly, samples were formalinfixed and paraffin-embedded. H\&E staining was used to confirm cancerous compartments. Cancer tissue with a diameter of at least $2 \mathrm{~mm}$ was chosen for immunohistochemistry staining, including ER, PR, Ki57, and p53. Immunohistochemistry was performed with an automated immunostainer (Leica Bond-Max, Milton Keynes, UK). Primary mouse monoclonal antibodies used in immunohistochemistry were ER (clone 1D5, 1:50), PR (clone PgR636, 1:500), Ki67 (clone MIB1, 1:300), and p53 (clone DO7, 1:200). The positive ratio of immunohistochemistry was calculated by positive tumor cells over total tumor cells per protocol. The positive rate of immunohistochemistry results was recorded as a percentage, such as Ki67 50\% $(+)$, ER 20\% (+), PR 20\% (+), and p53 (-), etc. Histological type, histological grade, lesion size, lesion-infiltration range, and immunohistochemistry results were initially judged by the primary pathologist, then reviewed by the senior physician.

\section{Statistical Analysis}

$\mathrm{R}$ software (version 3.6.1, package caret version 6.0-84, http://www.r-project.org)) was applied to split data randomly into an internal training set and an external validation set with a predefined ratio of 7:3. Splitting balance and consistency between the two data sets were tested to make sure the two cohorts were comparable. Categorical data were analyzed using $\chi^{2}$ and Fisher's exact tests, and continuous data using Student's $t$-tests and rank sum tests. $P<0.05$ was considered statistically significant.

The training cohort was applied to establish the model. Correlation of all factors with recurrence in EC was analyzed by univariate Cox regression. Those with $P<0.05$ were 
included in the multivariate Cox regression model and the HR of each factor calculated. Factors in multivariate regression with $P<0.05$ were selected to construct a nomogram model by R software to predict 1-, 3-, and 5-year RFS of EC patients. To identify the optimal threshold of the nomogram, a 3-year RFS rate for the patients was calculated and an ROC curve used. According to this optimal threshold, patients in the validation set were further divided into a highRFS group and a low-RFS group. Median follow-up and initial time of EC recurrence were calculated and compared. Kaplan-Meier analysis was used to determine the duration of RFS and overall survival rates in the two groups, and logrank tests were applied to determine significance.

In the validation cohort, performance of the prediction model was evaluated by bias-corrected calibration curves using Bootstrap version 1.3-23. The concordance index ( $\mathrm{C}$-index $)$ is a metric to evaluate predictions made by a model. ${ }^{15}$ Therefore, to test the efficacy of the proposed model further, $\mathrm{C}$-index values for three different models were calculated, including the model composed only of traditional indicators, the model composed only of immunohistochemical parameters, and the model proposed in our research. Besides R software, data were also analyzed using SPSS version 25.0 (IBM, Chicago, IL, USA).

\section{Results}

From October 2013 to May 2018, a total of 537 patients with stages I-III EC underwent primary surgical treatment at the First Affiliated Hospital of Chongqing Medical University, among which 473 meeting the criteria were recruited, while 54 were excluded, due to missing data, loss to follow-up, or nonstandard surgical approaches (Supplementary Figure). The six patients excluded were $<30$ years old and had stage IA EC. Because they required personalized surgical approaches for fertility preservation, oophorectomy was declined. Allcases were randomly divided into a training cohort $(\mathrm{n}=332,70 \%)$ and a validation cohort $(n=141,30 \%)$. Demographics and clinicopathological characteristics of women in the two cohorts are summarized in Table 1. Median follow-up for patients in the training cohort was 39 months, while that in the validation cohort was 35 months. There were 47 $(14.2 \%)$ recurrences in the training cohort, of which 26 patients died due to recurrence in EC and four due to other causes. Median RFS for relapsed patients was 12 months. Features between the two cohorts were comparable (Supplementary Table).

\section{Predictors of Recurrence in EC by Cox Regression Analysis}

We used univariate Cox regression analysis to analyze the clinicopathological factors and four immunohistochemical markers (Ki67, ER, PR, and p53) which might affect the recurrence of EC (Table 2). Factors with $P>0.05$ were excluded from multivariate analysis, including age ( $P=0.109)$, BMI $(P=0.653)$, hypertension $(P=0.664)$, diabetes $(P=0.291)$, and lymphadenectomy $(P=0.629)$. Factors with $P<0.05$, including FIGO stage, histological type and grade, myometrial invasion, cervical stromal invasion, adjuvant treatment, and all four immunohistochemical markers, were further included in the multivariate Cox regression. Finally, four factors with $P<0.05$ in multivariate regression analysis were recruited for modeling: FIGO stage, histological type, ER, and p53.

\section{Nomogram-Model Establishment and Evaluation}

A nomogram model was established (Figure 1) to calculate RFS in a more convenient and precise manner. The score of each predicting factor wasassigned to the first "Points" line, and then total points were added and assigned to 1-, 3-, and 5-year RFS. Calibration curves in both data sets showed good fitness between predictions and our data (Figure 2). In the comparison among different prediction models, discriminatory power demonstrated by C-index of our proposed model was 0.88 (95\% CI $0.84-0.92)$ in the training cohort and $0.79(95 \% \mathrm{CI} 0.66-0.92)$ in the validation cohort (Table 3).

\section{Optimal Threshold of Nomogram}

The optimal threshold of 3-year RFS in this model was 0.83 , determined by ROC curve with area under the curve 0.899 (95\% CI $0.852-0.946$ ), sensitivity $80.9 \%$, and specificity $87.0 \%$. Based on the optimal threshold, patients in the validation cohort with 3 -year RFS $>0.83$ were defined as the high-RFS group and low-RFS group. In the highRFS group, median follow-up and initial time of EC recurrence were 35 (range 7-67) and 23 (range 10-47) months, while for the low-RFS group these were 36 (range 7-63) and 11 (range 2-42) months, respectively. The 3-year RFS rates for the high-RFS group and low-RFS group were $96.6 \%(95 \%$ CI $92.7 \%-100 \%)$ and $57.4 \%$ (95\% CI 40.5\%-74.3\%, $P<0.001$; Figure 3A). The 3-year overall survival rates of the high-RFS group and low-RFS 
Table I Baseline Characteristics of the Training Cohort and Validation Cohort

\begin{tabular}{|c|c|c|c|c|c|}
\hline & Training Cohort, $n=332$ & $\%$ & Validation Cohort, $n=|4|$ & $\%$ & P-Value \\
\hline \multicolumn{6}{|l|}{ Age (years) } \\
\hline Median & 52 & & 54 & & 0.916 \\
\hline Range & $29-81$ & & 24-77 & & \\
\hline \multicolumn{6}{|l|}{ BMI $\left(\mathrm{kg} / \mathrm{m}^{2}\right)$} \\
\hline Median & 24.44 & & 24.65 & & 0.501 \\
\hline Range & $16.53-39.30$ & & $16.35-37.20$ & & \\
\hline Hypertension & 84 & 25.3 & 32 & 22.7 & 0.547 \\
\hline Diabetes & 53 & 16.0 & 15 & 10.6 & 0.131 \\
\hline \multicolumn{6}{|l|}{ FIGO stage } \\
\hline I & 245 & 73.8 & 102 & 72.3 & 0.834 \\
\hline II & 36 & 10.8 & 18 & 12.8 & \\
\hline III & 51 & 15.4 & 21 & 14.9 & \\
\hline \multicolumn{6}{|l|}{ Histological type } \\
\hline I & 287 & 86.4 & 121 & 85.8 & 0.856 \\
\hline II & 45 & 13.6 & 20 & 14.2 & \\
\hline \multicolumn{6}{|l|}{ Histological grade } \\
\hline 1 & 52 & 15.7 & 18 & 12.8 & 0.674 \\
\hline 2 & 185 & 55.7 & 79 & 56.0 & \\
\hline 3 & 95 & 28.6 & 44 & 31.2 & \\
\hline \multicolumn{6}{|l|}{ Myometrial invasion } \\
\hline$<1 / 2$ & 225 & 67.8 & 105 & 74.5 & 0.147 \\
\hline$\geq 1 / 2$ & 107 & 32.2 & 36 & 25.5 & \\
\hline Cervical stromal invasion & 63 & 19.0 & 26 & 18.4 & 0.891 \\
\hline Lymphadenectomy & 298 & 89.8 & 123 & 87.2 & 0.422 \\
\hline \multicolumn{6}{|l|}{ Adjuvant treatment } \\
\hline Follow-up or HT & 130 & 39.1 & 64 & 45.4 & 0.343 \\
\hline Only chemotherapy & 133 & 40.1 & 44 & 31.2 & \\
\hline Only radiotherapy & 10 & 3.0 & 5 & 3.5 & \\
\hline Chemoradiotherapy & 59 & 17.8 & 28 & 19.9 & \\
\hline \multicolumn{6}{|l|}{ Ki67-positive ratio (\%) } \\
\hline Median & 30 & & 30 & & 0.123 \\
\hline Range & $\mathrm{I}-90$ & & $0-90$ & & \\
\hline \multicolumn{6}{|l|}{ ER-positive ratio (\%) } \\
\hline Median & 80 & & 90 & & 0.684 \\
\hline Range & $0-95$ & & $0-95$ & & \\
\hline \multicolumn{6}{|l|}{ PR-positive ratio (\%) } \\
\hline Median & 80 & & 70 & & 0.087 \\
\hline Range & $0-95$ & & $0-95$ & & \\
\hline \multicolumn{6}{|l|}{ p53-positive ratio (\%) } \\
\hline Median & 20 & & 20 & & 0.948 \\
\hline Range & $0-95$ & & $0-90$ & & \\
\hline Recurrence & 47 & 14.2 & 20 & 14.2 & 0.994 \\
\hline \multicolumn{6}{|l|}{ Death } \\
\hline Death from EC & 26 & 7.8 & II & 7.8 & 1 \\
\hline Death from other disease & 4 & 1.2 & I & 0.7 & \\
\hline
\end{tabular}


Table I (Continued).

\begin{tabular}{|l|l|l|l|l|l|}
\hline & Training Cohort, $\mathbf{n = 3 3 2}$ & $\%$ & Validation Cohort, $\mathbf{n = 1 4 1}$ & $\%$ & $\mathbf{P}$-Value \\
\hline $\begin{array}{l}\text { Follow-up (months) } \\
\text { Median } \\
\text { Range }\end{array}$ & 39 & & 35 & \\
\end{tabular}

Abbreviations: BMI, body mass index; HT, hormonal treatment; FIGO, International Federation of Gynecology and Obstetrics; ER, estrogen receptor; PR, progesterone receptor.

Table 2 Univariate and Multivariate Analyses of Factors Predicting Endometrial Cancer Recurrence in the Training Cohort

\begin{tabular}{|c|c|c|c|c|c|c|}
\hline & \multicolumn{3}{|c|}{ Univariate Analysis } & \multicolumn{3}{|c|}{ Multivariate Analysis } \\
\hline & HR & $95 \% \mathrm{Cl}$ & P-Value & HR & $95 \% \mathrm{Cl}$ & P-Value \\
\hline \multicolumn{7}{|l|}{ FIGO stage } \\
\hline I & 1.000 & & $<0.001$ & 1.000 & & 0.014 \\
\hline II & 2.562 & $1.002-6.548$ & 0.049 & 1.626 & $0.423-6.248$ & 0.479 \\
\hline III & 7.017 & $3.792-12.985$ & $<0.001$ & 3.699 & $1.416-9.622$ & 0.008 \\
\hline Histological type (II vs I) & 8.000 & $4.509-14.193$ & $<0.001$ & 2.067 & $1.032-4.138$ & 0.040 \\
\hline \multicolumn{7}{|l|}{ Histological grade } \\
\hline I & 1.000 & & $<0.001$ & 1.000 & & 0.779 \\
\hline 2 & 5.249 & $0.703-39.211$ & 0.106 & 2.122 & $0.264-17.055$ & 0.479 \\
\hline 3 & 7.089 & $2.422-20.750$ & $<0.001$ & 2.094 & $0.24 I-18.203$ & 0.503 \\
\hline Myometrial invasion $(\geq I / 2$ vs $<I / 2)$ & 3.997 & $2.218-7.204$ & $<0.001$ & 1.450 & $0.689-3.050$ & 0.328 \\
\hline Cervical stromal invasion (Yes vs No) & 4.809 & $2.7 \mid 3-8.525$ & $<0.001$ & 1.434 & $0.579-3.554$ & 0.436 \\
\hline Adjuvant treatment (yes/o) & 4.037 & $1.808-9.014$ & 0.001 & 0.659 & $0.247-1.760$ & 0.406 \\
\hline Ki67-positive ratio & 1.034 & $1.020-1.047$ & $<0.001$ & 1.006 & $0.991-1.022$ & 0.407 \\
\hline ER-positive ratio & 0.968 & $0.958-0.977$ & $<0.001$ & 0.984 & $0.970-0.998$ & 0.028 \\
\hline PR-positive ratio & 0.972 & $0.964-0.981$ & $<0.001$ & 0.999 & $0.984-1.014$ & 0.868 \\
\hline p53-positive ratio & 1.020 & $1.011-1.028$ & $<0.001$ & 1.010 & $1.001-1.020$ & 0.032 \\
\hline
\end{tabular}

Abbreviations: FIGO, International Federation of Gynecology and Obstetrics; ER, estrogen receptor; PR, progesterone receptor.

group were $97.6 \%(95 \%$ CI $94.3 \%-100 \%)$ and $69.9 \%$ (95\% CI 54.2\%-85.6\%, $P<0.001$; Figure 3B).

\section{Discussion}

In this study, four immunohistochemical markers (Ki67, $\mathrm{ER}, \mathrm{PR}$, and p53) were analyzed, which are also commonly used in clinical practice, and two (ER and p53) were included in the model. Using this nomogram model, the postoperative recurrence of each EC patient with FIGO stages I-III was able to be predicted in a more precise and convenient manner. Firstly, including immunohistochemical markers in this model increased the discrimination of recurrence risk among EC patients. It has been demonstrated by the internal and external calibration (Figure 2) and the comparison among different models (Table 3 ) that this proposed model had the highest prediction accuracy and consistency compared to traditional models or immunohistochemical markers only. Secondly, the use a nomogram makes this model a convenient tool for clinical use. For example, if a patient had FIGO stage II (42 points), histological type I (0 points), ER 20\% (+, 80 points), and P53 70\% (+, 40 points), she would get a total score of 162 points, corresponding to a 3-year RFS rate of $70 \%$ (recurrence probability in three years is $30 \%$ ). As such, this proposed model can explain the abstract concept of postoperative recurrence in quantitative form instead of a simple conclusion, as "high risk" or "low risk", for recurrence based on several predictors. Furthermore, the results showed that both RFS and overall survival of the low-RFS group were much lower than the high-RFS group. Therefore, the model was able to stratify EC patients according to risk of recurrence, and it also suggested that the postoperative management of the lowRFS group should have more attention to it: for patients 
Points

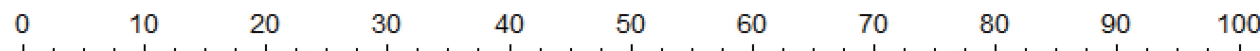

FIGO stage

Histologic type

$E R(\%)$

P53(\%)

Total Points

II

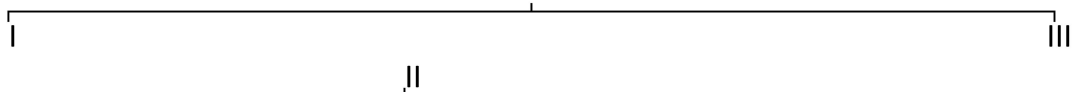

II

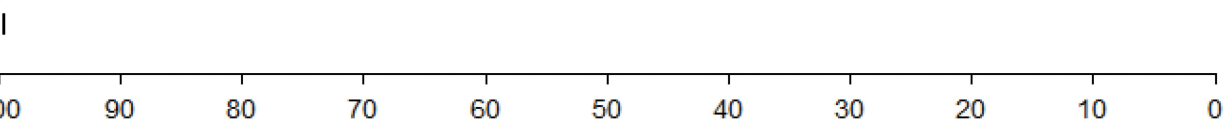

\begin{tabular}{lllllllllll}
\hline 0 & 10 & 20 & 30 & 40 & 50 & 60 & 70 & 80 & 90 & 100
\end{tabular}

Linear Predictor

\begin{tabular}{|c|c|c|c|c|c|}
\hline & 50 & 100 & 150 & 200 & 250 \\
\hline
\end{tabular}

1 year RFS

\begin{tabular}{|c|c|c|c|c|c|c|c|c|c|c|c|}
\hline$\Gamma$ & 1 & $T$ & 1 & $T$ & $T$ & $T$ & 1 & $T$ & $T$ & $T$ & \\
\hline-1.5 & -1 & -0.5 & 0 & 0.5 & 1 & 1.5 & 2 & 2.5 & 3 & 3.5 & 4 \\
\hline
\end{tabular}

3 year RFS

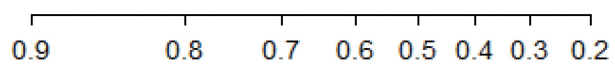

5 year RFS

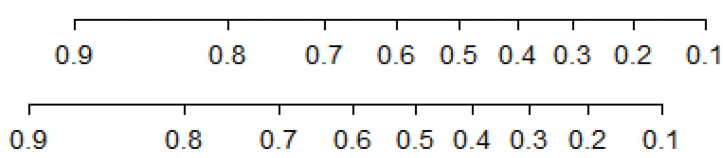

Figure I Nomogram model for estimating the rate of recurrence-free survival (I, 3, or 5 years) for women with FIGO stage I-III endometrial cancer.

Notes: To estimate recurrence risk, calculate points for each variable by drawing a straight line from patient-variable value from the second to the fifth to the first line, labeled "Points". Sum all points, and draw a straight line from the sixth to the eighth to the tenth line to get I-, 3-, and 5-year recurrence-free survival rates.
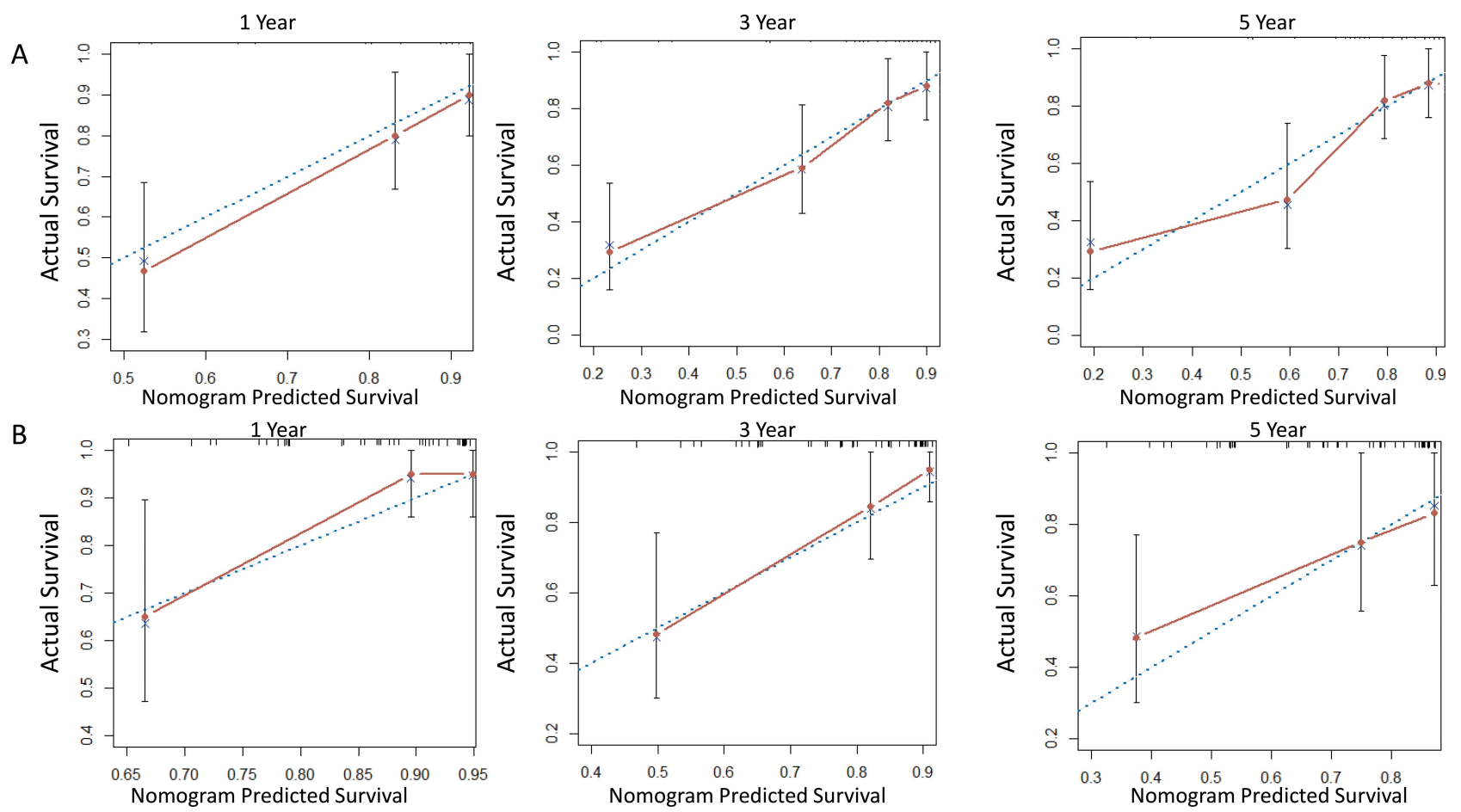

Figure 2 (A) Calibration curve for internal validation of the nomogram model for predicting RFS in EC; (B) calibration curve for external validation of the nomogram model for predicting RFS in EC.

Notes: (A, B) Blue dotted line, reference; solid red line, prediction curve given by the model.

who would receive postoperative adjuvant treatment (chemotherapy and/or radiotherapy), cycles of postoperative adjuvant therapy should be increased appropriately compared with the original basis, and follow-up of them should be closer, while for some early low-risk EC patients who would not receive adjuvant therapy after 

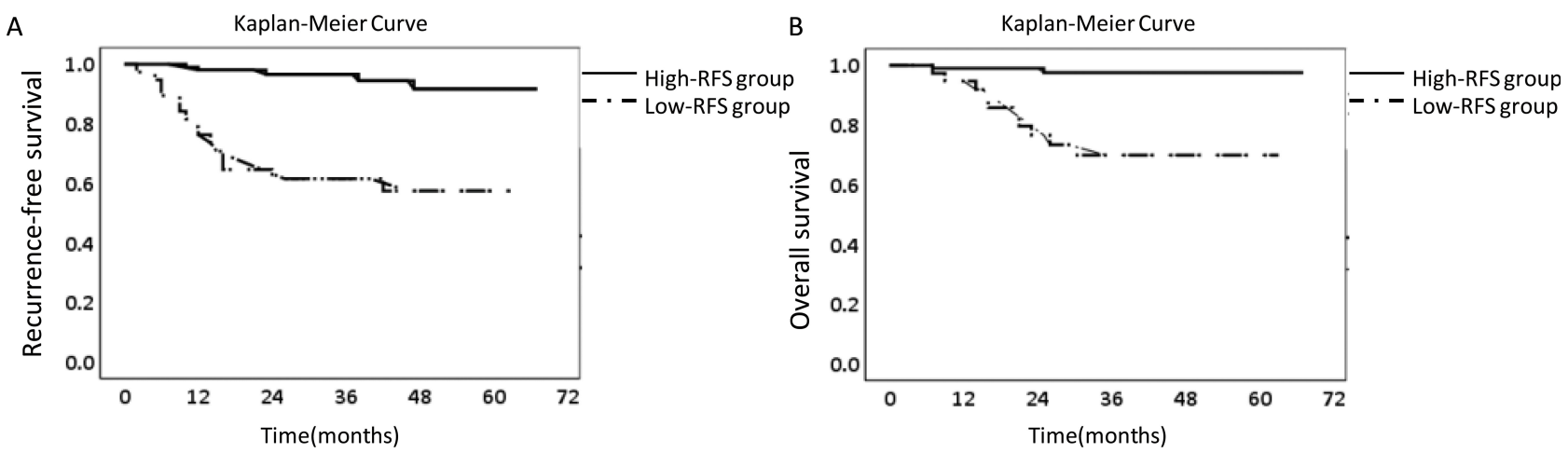

Figure 3 (A) Recurrence-free survival according to nomogram optimal threshold; (B) overall survival according to nomogram optimal threshold. Notes: (A, B) Dotted line, recurrence-free survival or overall survival for low-RFS group patients in validation cohort; solid line, recurrence-free survival or overall survival for high-RFS group patients in validation cohort.

surgery, the appropriate postoperative adjuvant treatment still might be necessary.

In recent years, Cancer Genome Atlas molecular subgroups, including POLE, MSI, copy-number high, and copy-number low, have shown increasing potential value in EC prognosis. ${ }^{16-18}$ Many studies have reported that there are obvious different EC prognoses among the four subgroups. ${ }^{16}$ Prognosis of the copy number-high group is the worst, and easily deteriorate, further, due to adverse clinicopathological factors. Secondly, prognosis of the MSI group is similar to the copy number-low group, but can still easily deteriorate, due to adverse clinicopathological factors. Prognosis of the POLE group is best, and it does not seem to be significantly affected by clinicopathological factors. However, such novel classifications have not been widely used in clinical practice, because of the high cost of sequencing and technical difficulties in many regions, while immunohistochemistry, having been applied for decades, is still an important component of the present postoperative histopathological report, as it is simple, inexpensive, and less time-consuming.

Table 3 The Discriminatory Power (C-Index) for RecurrenceFree Survival in the Training Cohort and Validation Cohort

\begin{tabular}{|c|l|l|l|l|}
\hline \multirow{2}{*}{ Combinations } & \multicolumn{2}{|l|}{ Training Cohort } & \multicolumn{2}{l|}{ Validation Cohort } \\
\cline { 2 - 5 } & C-Index & $\mathbf{9 5 \%} \mathbf{~ C l}$ & C-Index & $\mathbf{9 5 \% ~ C l ~}$ \\
\hline $\begin{array}{l}\text { FIGO, His-type, } \\
\text { His-grade, cervical } \\
\text { stromal invasion } \\
\text { ER, PR, p53 }\end{array}$ & 0.84 & $0.78-0.90$ & 0.76 & $0.63-0.89$ \\
FIGO, His-type, \\
ER, p53
\end{tabular}

Abbreviations: FIGO, International Federation of Gynecology and Obstetrics; His-, histological; ER, estrogen receptor; PR, progesterone receptor.
However, some points should be clarified before this model is applied. Though only four factors were included in this model, other factors, such as histological grade, depth of myometrial invasion, cervical stromal infiltration, Ki67, or PR, have been shownto have prognostic importance in EC. ${ }^{4,5,19}$ According to the risk ratio of the univariate analysis, it seemed that EC was more likely to relapse in patients who had received adjuvant therapy. This could have been confounded by adjuvant therapy being administered to patients at late stages or other high-risk factors for recurrence, which caused adjuvant therapy as a prognostic factor to have strong "collinearity" on univariate analysis, ${ }^{20}$ and the "protective effect" of adjuvant therapy may not be adequate to offset the risk raised by these risk factors. ${ }^{21,22}$ In this study, the 3-year RFS rate of patients was predicted. Though the follow-up of a small proportion of patients (about $20 \%$ of the total patients;proportion of censored data within the statistically allowable range ${ }^{23}$ ) was $<3$ years, the overall median follow-up of patients was about 3 years. Also, median RFS time of relapsed patients was concentrated at 12 months, and except for some patients whose follow-up did not reach 1 year due to death, the remaining patients' follow-up was all $>1$ year. Therefore, this study ensured that there was sufficient follow-up for patients.

Given the number of cases recruited, this model could have been optimized in the following three aspects. Firstly, besides the four immunohistochemical markers included in this study, markers like serum Ca125, CDK4/6, cytotoxic T cells, memory T cells, and L1CAM have demonstrated potential. ${ }^{24-27}$ It is possible that more markers could contribute to a better prediction model. Another issue is that this model included patients with 
FIGO stage III like previous studies, ${ }^{4,28,29}$ but it is likely that more importance should be attached to predict recurrence in early-stage EC and thus facilitate the prescription of personalized treatment schemes for those patients. Therefore, we are going to recruit more patients with early-stage EC to establish new models in future. Finally, this model was established from a retrospective and single-center study, though a training cohort and validation cohort were set to minimize the bias. The model requires multicenter data to test its universality further. In summary, we have developed a nomogram model for predicting 1-, 3-, and 5-year recurrence-free survival of patients with FIGO stages I-III EC. This convenient and quantifiable tool can facilitate the treatment and follow-up of patients with EC.

\section{Abbreviations}

EC, endometrial cancer; ER, estrogen receptor; PR, progesterone receptor; RFS, recurrence-free survival; OS, overall survival; FIGO, International Federation of Gynecology and Obstetrics; ROC, receiver-operating characteristic.

\section{Data-Sharing Statement}

The data sets used and/or analyzed during the current study are available from the corresponding author on reasonable request.

\section{Ethics Approval and Consent to Participate}

The study was approved by Ethics Committee of Chongqing Medical University and conducted in accordance with the Declaration of Helsinki. All patients provided informed consent before starting treatment. As it was a retrospective clinical study, all patients were contacted by telephone to obtain verbal informed consent, which was approved by the Ethics Committee. All data on the patients was anonymized or maintained with confidentiality.

\section{Disclosure}

The authors report no conflicts of interest for this work.

\section{References}

1. Colombo N, Creutzberg C, Amant F, et al. ESMO-ESGO-ESTRO Consensus Conference on Endometrial Cancer: diagnosis, treatment and follow-up. Ann Oncol. 2016;27(1):16-41. doi:10.1093/annonc/ $\operatorname{mdv} 484$
2. Creasman WT, Odicino F, Maisonneuve P, et al. Carcinoma of the Corpus Uteri. Int $J$ Gynecol Obstetrics. 2006;95:S105-S43. doi:10.1016/S0020-7292(06)60031-3

3. Sagae S, Susumu N, Viswanathan AN, et al. Gynecologic Cancer InterGroup (GCIG) consensus review for uterine serous carcinoma. Int J Gynecol Cancer. 2014;24(9 Suppl 3):S83-9. doi:10.1097/ IGC.0000000000000264

4. Ouldamer L, Bendifallah S, Body G, et al. Predicting poor prognosis recurrence in women with endometrial cancer: a nomogram developed by the FRANCOGYN study group. Br J Cancer. 2016;115 (11):1296-1303. doi:10.1038/bjc.2016.337

5. Takahashi K, Yunokawa M, Sasada S, et al. A novel prediction score for predicting the baseline risk of recurrence of stage I-II endometrial carcinoma. J Gynecol Oncol. 2019;30(1):e8. doi:10.3802/ jgo.2019.30.e8

6. Gulseren V, Kocaer M, Ozdemir IA, Cakir I, Sanci M, Gungorduk K. Do estrogen, progesterone, P53 and Ki67 receptor ratios determined from curettage materials in endometrioid-type endometrial carcinoma predict lymph node metastasis? Curr Probl Cancer. 2020;44 (1):100498. doi:10.1016/j.currproblcancer.2019.07.003

7. Ohara M, Matsuura K, Akimoto E, et al. Prognostic value of Ki67 and $\mathrm{p} 53$ in patients with estrogen receptor-positive and human epidermal growth factor receptor 2-negative breast cancer: validation of the cut-off value of the Ki67 labeling index as a predictive factor. $\mathrm{Mol}$ clin oncol. 2016;Apr(4):648-654.

8. Abubakar M, Figueroa J, Ali HR, et al. Combined quantitative measures of ER, PR, HER2, and KI67 provide more prognostic information than categorical combinations in luminal breast cancer. Mod Pathol. 2019;32(9):1244-1256. doi:10.1038/s41379-019-0270-4

9. Oncology FCoG. FIGO staging for carcinoma of the vulva, cervix, and corpus uteri. Int $J$ Gynaecol Obstet. 2014;125(2):97-98. doi:10.1016/j.ijgo.2014.02.003

10. Marcos-Sanmartin J, Lopez Fernandez JA, Sanchez-Paya J, et al. Does the type of surgical approach and the use of uterine manipulators influence the disease-free survival and recurrence rates in early-stage endometrial cancer? Int J Gynecol Cancer. 2016;26 (9):1722-1726. doi:10.1097/IGC.0000000000000808

11. Colombo NPE, Landoni F, Carinelli S, Colombo A, Marini C, Sessa C, ESMO Guidelines Working Group. Endometrial cancer: ESMO clinical practice guidelines for diagnosis, treatment and follow-up. Ann Oncol. 2013;24(Suppl 6):vi33-vi38. doi:10.1093/ annonc/mdt353

12. Ouldamer L, Bendifallah S, Body G, et al. Change in hazard rates of recurrence over time following diagnosis of endometrial cancer: an age stratified multicentre study from the FRANCOGYN group. Eur $j$ Surg Oncol. 2018;44(12):1914-1920. doi:10.1016/j.ejso.2018.07.053

13. Huijgens ANJ. Factors predicting recurrent endometrial cancer. $F V V$ ObGyn. 2013;5(3):179-186.

14. Yu X, Guo S, Song W, et al. Estrogen Receptor $\alpha(\mathrm{ER} \alpha)$ status evaluation using RNAscope in situ hybridization: a reliable and complementary method for IHC in breast cancer tissues. Hum Pathol. 2017;61:121-129. doi:10.1016/j.humpath.2016.12.005

15. Brentnall AR, Cuzick J. Use of the concordance index for predictors of censored survival data. Stat Methods Med Res. 2018;27 (8):2359-2373. doi:10.1177/0962280216680245

16. Raffone A, Travaglino A, Mascolo M, et al. TCGA molecular groups of endometrial cancer: pooled data about prognosis. Gynecol Oncol. 2019;155(2):374-383. doi:10.1016/j.ygyno.2019.08.019

17. Leon-Castillo A, Gilvazquez E, Nout R, et al. Clinicopathological and molecular characterisation of 'multiple-classifier' endometrial carcinomas. J Pathol. 2020;250(3):312-322. doi:10.1002/path.5373

18. Guo S, Yang J, Wu M, Xiao G. Clinical value screening, prognostic significance and key pathway identification of miR-204-5p in endometrial carcinoma: a study based on the Cancer Genome Atlas (TCGA), and bioinformatics analysis. Pathol Res Pract. 2019;215 (5):1003-1011. doi:10.1016/j.prp.2019.02.007 
19. Ferrandina G, Ranelletti FO, Gallotta V, et al. Expression of cyclooxygenase-2 (COX-2), receptors for estrogen (ER), and progesterone (PR), p53, ki67, and neu protein in endometrial cancer. Gynecol Oncol. 2005;98(3):383-389. doi:10.1016/j.ygyno.2005.04.024

20. Pierre-Graud Claret XB, de La Coussaye JE. Collinearity and multivariable analysis. Intensive Care Med. 2016;42(11):1834 doi:10.1007/s00134-016-4528-8

21. Alessia Aloisi FP, Scaletta G, Capriglione S, et al. Chemotherapy as adjuvant treatment for intermediate-high risk early-stage endometrial cancer: a pilot study. Int J Gynecol Cancer. 2015;25(8):1418-1423. doi:10.1097/IGC.0000000000000505

22. Emons G, Vordermark D. Adjuvant treatment for endometrial cancer Curr Opin Oncol. 2019;31(5):404 410. doi:10.1097/CCO.00000 00000000558

23. Xue X, Xie X, Strickler HD. A censored quantile regression approach for the analysis of time to event data. Stat Methods Med Res. 2018;27 (3):955-965. doi:10.1177/0962280216648724

24. van der Putten LJM, Visser NCM, van de Vijver K, et al. Added value of estrogen receptor, progesterone receptor, and L1 cell adhesion molecule expression to histology-based endometrial carcinoma recurrence prediction models: an ENITEC Collaboration Study. Int J Gynecol Cancer. 2018;28(3):514-523. doi:10.1097/IGC.00 00000000001187
25. Versluis MA, de Jong RA, Plat A, et al. Prediction model for regional or distant recurrence in endometrial cancer based on classical pathological and immunological parameters. $\mathrm{Br} J$ Cancer. 2015;113 (5):786-793. doi:10.1038/bjc.2015.268

26. Nakamura K, Imafuku N, Nishida $T$, et al. Measurement of the minimum apparent diffusion coefficient (ADCmin) of the primary tumor and CA125 are predictive of disease recurrence for patients with endometrial cancer. Gynecol Oncol. 2012;124(2):335-339. doi:10.1016/j.ygyno.2011.10.014

27. Ikeda Y, Oda K, Ishihara H, et al. Prognostic importance of CDK4/ 6-specific activity as a predictive marker for recurrence in patients with endometrial cancer, with or without adjuvant chemotherapy. $\mathrm{Br}$ $J$ Cancer. 2015;113(10):1477-1483. doi:10.1038/bjc.2015.369

28. Smith D, Stewart CJR, Clarke EM, et al. ER and PR expression and survival after endometrial cancer. Gynecol Oncol. 2018;148 (2):258-266. doi:10.1016/j.ygyno.2017.11.027

29. Violante Di Donato VI, Schiavi MC, Colagiovanni V, et al. Impact of hormone receptor status and $\mathrm{Ki}-67$ expression on disease-free survival in patients affected by high-risk endometrial cancer. Int J Gynecol Cancer. 2018;28(3):505-513. doi:10.1097/IGC.0000000000001191

\section{Publish your work in this journal}

Cancer Management and Research is an international, peer-reviewed open access journal focusing on cancer research and the optimal use of preventative and integrated treatment interventions to achieve improved outcomes, enhanced survival and quality of life for the cancer patient.
The manuscript management system is completely online and includes a very quick and fair peer-review system, which is all easy to use. Visit http://www.dovepress.com/testimonials.php to read real quotes from published authors. 